SPATIAL COGNITION AND COMPUTATION, vol(issue), 1-end

Copyright (C) YYYY, Lawrence Erlbaum Associates, Inc.

\title{
A Hybrid Semantic Similarity Measure for Spatial Information Retrieval
}

\author{
Angela Schwering \\ University of Osnabrück \\ Werner Kuhn \\ University of Münster
}

Correspondence concerning this article should be addressed to Angela Schwering, University of Osnabrück; email aschweri@uos.de. 


\title{
2 SCHWERING, KUHN
}

\begin{abstract}
Semantic similarity is central to many cognitive processes and plays an important role in the way humans process and reason about information. In particular, the retrieval of knowledge from memory hinges crucially on similarity. Likewise, information retrieval systems use similarity to detect relevant information for a given query. Current information retrieval systems apply mainly syntactic techniques to determine similarity. Although such syntactic similarity measures have performed strongly with resources containing large amounts of text, they cannot appropriately cope with syntactic and semantic heterogeneity and ambiguity, if the semantics of the terms is not explicitly available. Therefore, they are rather rigid and inflexible as they cannot adapt to the user's requirements and conceptualization of the domain. Furthermore, geographic features are distinguished via their geometric and thematic data. It is often not possible to capture the complex semantics of geographic features by a single name or a textual description. Therefore spatial data is different from text documents typically found in enterprise databases or on the Web. Retrieval of spatial information requires new, intelligent retrieval mechanisms that satisfy its specific requirements. A semantics-based solution can more easily adapt to user needs and therefore increases the flexibility and usability of spatial data and retrieval methods. This paper investigates the suitability of various approaches - originally developed to explain human similarity judgement - in the context of spatial information retrieval. We propose a new, hybrid approach for semantic similarity measurement, which can represent the complex semantics of spatial data. It allows for retrieving relevant data by determining the similarity between the query and the semantic descriptions of geographic feature types within the database. The hybrid similarity measure combines the geometric structure of conceptual spaces with the relational structure of semantic nets to one, cognitively plausible knowledge representation with an inherent similarity measure.
\end{abstract}

Keywords: Spatial Information Retrieval, Semantic Similarity Measurement, Cognitive Semantics

\section{Introduction}

Semantic similarity is a central construct of human thinking and reasoning. For example, humans use similarity to organize their knowledge, assign objects and events to specific categories and form general concepts with certain attributes and behavior (Goldstone \& Son, 2005). Unfamiliar objects are recognized based on their similarity to previous knowledge. New problems are solved by applying solutions of similar problems. Many cognitive abilities hinge crucially on simi- 
A HYBRID SIMILARITY MEASURE 3

larity, and therefore similarity should play an important role also for the design of information systems.

Information retrieval systems organize and manage large amounts of information and make it accessible for users. Like humans when they retrieve knowledge from memory, information retrieval systems can apply similarity measures to determine the relevance of information with respect to a certain user query. Stateof-the-art retrieval systems use mainly syntactic similarity measures, which have performed strongly with textual information, the predominant kind of information in enterprises or on the web. However, the existing syntactic search strategies have disadvantages with other kinds of content such as spatial data. Spatial information is carried by geometric and thematic data. Feature catalogues often provide a textual description of a feature type, but this is insufficient to capture its complex semantics. Syntactic similarity measures cannot assess the relevance of information based on its content. Problems occur when syntactic similarity measures deal with heterogeneous or ambiguous data: often queries contain search terms which are not recorded in the database or terms which have different meanings in the query and in the database. Semantic information retrieval mechanisms compare the meaning specified for queries and data, search for information with similar meanings and retrieve information based on relevance.

This paper presents semantic similarity measures for spatial data in the context of an information retrieval task and proposes a new hybrid approach which combines geometric and network representations. It is based on the premise that a retrieval system should reflect human retrieval strategies. Therefore, our research focuses on psychologically motivated approaches which process semantics of spatial data in a cognitively plausible way. The remainder of the paper is structured as follows: after briefly introducing the case study in section 2 , we give an overview over similarity measures developed as psychological approaches to model human similarity judgements in section 3 and evaluate them with respect to spatial information retrieval. In the subsequent two sections we describe the hybrid similarity measure: section 4 explains the knowledge representation and outlines how spatial semantics can be captured; section 5 describes the actual procedure to determine the similarity between two spatial concepts ${ }^{1}$. In section 6 we evaluate our approach and compare it to the existing similarity measures. Finally, we summarize the paper and give conclusions and directions for future work.

\footnotetext{
${ }^{1}$ In his paper, we use the term "concept" to stand for a human conceptualization of a class of individuals. An equally good (as well as equally disputed) term would have been "category". For conceptualizations of individuals, we use the term "individual".
} 


\section{SCHWERING, KUHN}

\section{Case Study}

The proposed hybrid similarity measure is based on semantic descriptions and therefore is suitable for different types of data. In this paper we focus on a geospatial case study and the particular requirements of spatial data.

Due to more frequent floodings of land in recent times, flood risk and defence management has become a major topic for applications of spatial information. Canalization, straightening of rivers, and bank reinforcement lead to the problem that water cannot spread out after snowmelt and heavy rainfall. Destruction of wetlands, decommissioning of rain storage reservoirs, and the loss of flood plains further increase the danger of flooding. To provide reliable flood warnings, data about topography, hydrology and geology is needed. Although this data is available in various sources, it is often difficult to find relevant data and make it usable for a specific context. Our case study is centered around a retrieval task to find relevant data for flood risk management, in particular information about flooding areas. We show that semantic information retrieval can find relevant information about flooding areas, even if these are not explicitly specified as such.

\section{Psychologically Motivated Similarity Measures}

We summarize different kinds of similarity measures, focussing on models originally developed to explain or predict human similarity perception. All these models have been tested in experiments of human similarity judgement. A detailed overview can be found in (Schwering, 2008).

\subsection{Geometric Models}

Geometric models of knowledge representation were first used in psychology to exploit the analogy to space for measuring similarity (Attneave, 1950; Torgerson, 1958, 1965). Concepts are modeled within a multidimensional space and their spatial distance is an indicator of semantic similarity. Each dimension or set of dimensions of this multidimensional space represents one quality (e.g. color) and concepts are described by applicable values on quality dimensions. Figure 1 illustrates a space for the concept flooding area. Concepts are represented by an $\mathrm{n}$-dimensional region in the vector space and individuals by a point. Properties are represented as values on dimensions, e.g. flat and gentle slope are different values on the slope dimension.

Similarity is measured in analogy to spatial proximity: The spatial distance in the vector space is interpreted as the semantic distance. The nearer two concepts are, the smaller is their semantic distance and the higher their similarity. The most prominent distance measures in vector spaces are the Minkowski metrics. They are represented by a generic formula (equation 1): $r=1$ results in the city- 
A HYBRID SIMILARITY MEASURE 5

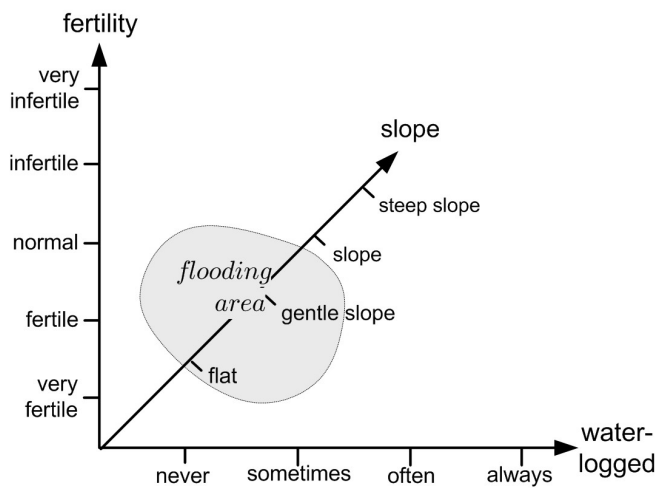

Figure 1: Geometric model represent concepts in vector spaces. The concept flooding area is modeled in a three-dimensional conceptual space.

block distance and $r=2$ in the Euclidian distance (Suppes, Krantz, Luce, \& Tversky, 1989). Similarity is then a decaying function of semantic distance $d(q, c)$ (Attneave, 1950; Melara, Marks, \& Lesko, 1992; Shepard, 1957, 1958a, 1958b).

$$
d(q, c)=\left[\sum_{i=1}^{n}\left|q_{i}-c_{i}\right|^{r}\right]^{1 / r}
$$

A core characteristic and at the same time the most heavily criticized aspect of geometric similarity models are their metric assumptions (Johannesson, 2002): Similarity satisfies the three metric axioms minimality (the semantic distance to itself is always zero), symmetry (the similarity from $a$ to $b$ is the same as from $b$ to $a$ ), and triangle inequality (the semantic distance from $a$ to $b$ plus the semantic distance from $b$ to $c$ is greater than the direct semantic distance from $a$ to $c$ ).

\subsection{Feature Models}

Feature models are based on set theory. They have been used to discover the structure of semantic memory (Meyer, 1970; Schaeffer \& Wallace, 1970) and to analyze cognitive processes. The most prominent feature models are Tversky's contrast and ratio models $(1987,1977,1978,1982)$. Individuals are represented via an unstructured set of features (figure 2).

The contrast model (equation 2) and the ratio model (equation 3 ) assess the similarity of two individuals via their ratio of common $(A \cap B)$ and distinct $(A-B$ and $B-A$ ) features. While common features increase the similarity, distinct features decrease it. This similarity measure is asymmetric and satisfies neither minimality nor the triangle inequality (if $\alpha \neq \beta$ ). 


\section{SCHWERING, KUHN}

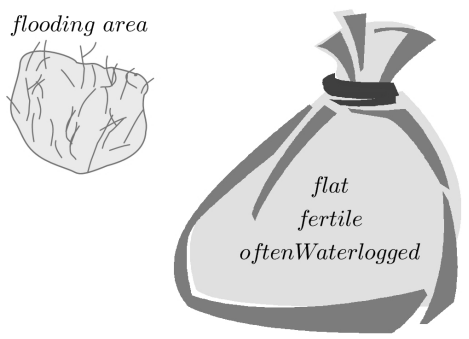

Figure 2: Feature models represent concepts by an unstructured set of features.

$$
\begin{aligned}
& S(a, b)=\theta * f(A \cap B)-\alpha * f(A-B)-\beta * f(B-A) \\
& S(a, b)=\frac{f(A \cap B)}{\theta * f(A \cap B)+\alpha * f(A-B)+\beta * f(B-A)}
\end{aligned}
$$

Feature models assess only entire feature matches and cannot detect graded similarity between features, i.e. compound features such as sometimesWaterlogged and oftenWaterlogged do not match and are as dissimilar as sometimesWaterlogged and neverWaterlogged. In contrast to the geometric model, feature models cannot detect intra-dimensional feature similarity.

\subsection{Network Models}

Network models are built upon semantic nets which have their origins in psychology where they have been used to model human semantic memory. Knowledge about the world can be structured in a network comprising concepts connected through appropriate relationships. Collins and Quillian (1969) gave the first empirical evidence of semantic networks in their 1969 experiment about how humans store information.

Network models describe semantics of concepts indirectly by relating them to other concepts. A concept flooding area could be described by a semantic net as shown in figure 3. Similarity in network models is computed based on the shortest path in a network. Various approaches exist to measure similarity in networks: In Rada et al. (1989), a function was proposed to compute similarity based on the distance between concepts in a semantic net. Rodriguez et al. (2000, 2003a) propose a semantic similarity measure based on the feature similarity of a concept's semantic neighborhood. A semantic neighborhood of a concept $c$ is defined as the set of concepts whose network distances to $c$ are equal to or less than $r$ which is the radius of the neighborhood. 
A HYBRID SIMILARITY MEASURE 7

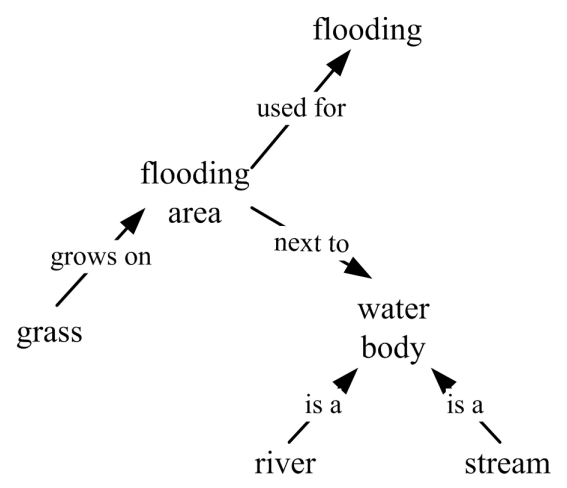

Figure 3: Network models represent concepts by relations to other concepts.

\subsection{Alignment Models}

The notion of alignment for knowledge representations and similarity measurement arose from studies on structural alignment and mapping in analogy and similarity by Gentner and Markman (1994, 1995). For a long time analogy was seen as a sophisticated process involving structural alignment of mental representations, while similarity was regarded as a primitive, purely perceptual cognitive process. Gentner et al. examined human similarity assessment and showed that commonalities of the relational structures are equally important for similarity judgement (1985, 1993). By the slogan "similarity is like analogy" (1995, p.114), Gentner et al. asserted that human similarity assessment also involves structural alignment and mapping. While feature, geometric, and network models are unstructured knowledge representations, alignment models are the first to represent knowledge in a structured way, i.e. alignment models represent concepts with their parts, describe parts separately with features, relate parts to wholes, and relate concepts to other concepts.

Goldstone et al. (1994, 1994) introduced the "Similarity, Interactive Activation, and Mapping" (SIAM) model, an alignment model for similarity measurement (figure 4). It is mainly used for similarity measurement between spatial scenes. The scenes are described by roles, components and features. Figure 4 shows a spatial scene with a floodplain and a river and a description of this scene in SIAM. Relations describe hierarchical or propositional representations of individuals, such as the spatial relation in the example. SIAM computes all possible alignments of features and relations. All correspondences must be structurally consistent, which involves two constraints: one-to-one mapping and parallel connectivity (Markman, 1999). One-to-one mapping means that each element is mapped to exactly one element. Parallel connectivity means that the arguments of corresponding 


\section{SCHWERING, KUHN}

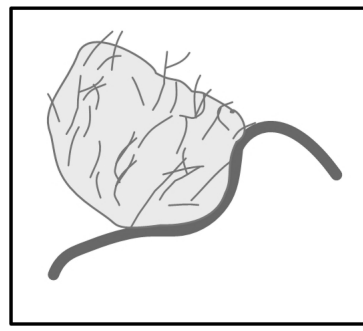

\section{Representation:}

above and left [

(flooding area: slope flat, waterlogged sometimes),

(waterbody: slope gentle_slope, waterlogged always)]

Figure 4: SIAM, the alignment model proposed by Goldstone, describes spatial scenes in a structured way by their components, the role they play, and their properties.

roles must also correspond. SIAM iteratively revises inconsistent alignments until it is consistent and similarity can be determined (Goldstone, 1994).

\subsection{Transformational Models}

All similarity models presented so far describe the concepts according to their properties and relations and measure similarity based on the comparison of these descriptions. Transformational models, instead, describe concepts indirectly by defining a minimal set of transformations required to distort one concept into another. Similarity between these two concepts is computed in terms of the number or the complexity of transformations needed to make concepts equal.

Transformational models provide a straight forward approach to determine the similarity between two concepts. The main problem is to find suitable sets of transformations: The transformational model has been frequently applied in welldefined domains of perceptual stimuli, for example symbol chains such as alphabetic strings (Wiener-Ehrlich, Bart, \& Millward, 1980), chains of filled and unfilled circles (Imai, 1977) or geometric complexes (Hahn, Richardson, \& Chater, 2003). The transformations focus on the perceptual attributes only. Operations such as mirror, reverse, add symbols are used to modify the order of symbol chains. Operations modifying the geometric arrangement are rotation, reflection, translation and dilation (Goldstone \& Son, 2005). However, these perceptual transformations are not sufficient to describe the relation of conceptual stimuli and it remains unclear how a set of transformations might look for geo-spatial concepts.

\subsection{Logic-based Similarity Models}

The above-mentioned similarity measures have all developed from psychological models for human similarity judgment. Another important group of computa- 
A HYBRID SIMILARITY MEASURE 9

tional similarity measures are logic-based approaches. They have gained a more and more important role in semantic interoperability research, especially through the increased development of formal ontologies. Janowicz (2006a, 2006b) has proposed SIM-DL, a similarity measure for geo-spatial ontologies. Concepts are specified based on primitive concepts, roles and language constructors such as intersection, union, and existential quantification. Similarity is measured by determining the overlap of a query concept definition and concept definitions in an ontology. SIM-DL is an asymmetric and context aware similarity measure which can be used for information retrieval.

Since logic-based similarity measures play an important role in knowledge representation and reasoning we include them in the overview of related work. However, the hybrid similarity measure was mainly inspired by ideas of similarity measures originally developed as psychological approaches to model human similarity judgements.

\subsection{Shortcomings with Respect to Spatial Data}

Five of the six presented similarity models were developed to explain human similarity judgement, but each has a different mathematical foundation, requires a different knowledge representation and implies a different notion of similarity. With respect to spatial data, it is important that the similarity measure can account for complex semantics: the similarity measure has to model properties as well as (spatial) relations and should provide a structured similarity measure. Furthermore, the approaches must support similarity at a conceptual level: We do not compare single instances of geometric features, but conceptual descriptions of feature types.

Geometric models use dimensional properties to specify semantics. Relations can only be represented as "compound dimensions", e.g. flooding area would have the value yes on the compound dimension nextToWater Body. In (Schwering \& Raubal, 2005) an extension was proposed to model relations as dimensions. However, the disadvantage of loosing the relational structure remains. Therefore geometric models do not allow for structured representation of concepts, involving, for example, hierarchic or part-whole relations, nor for representing the scope of properties, e.g. properties referring only to a part of a concept (Schwering, 2005).

The same disadvantage holds for feature models: two individuals cannot be related in a structured way, e.g. the fact that flooding areas typically lie next to water bodies can again only be expressed by the compound feature nextToWaterBody. No partial match would be detected between nextToWaterBody and nextToRiver. A relational representation of these features - e.g. nextTo(flooding area,waterbody) and nextTo(flooding area, river) - could detect similarity via an alignment of ar- 


\section{SCHWERING, KUHN}

guments. Like geometric models, feature models do not support a structured representation of concepts (Schwering, 2005).

The strength of the network approach is the representation of relations between concepts. Pure network models do not describe concepts any further (e.g. by features or dimensions) though they can be combined with feature models such as the semantic neighborhoods in the Matching-Distance Similarity Model (Rodriguez \& Egenhofer, 2003b, 2004).

Alignment models describe the structure of individuals by specifying their properties and the relations between their parts. However, they were developed to measure the similarity between individuals such as spatial scenes. SIAM provides good results, if the individuals compared have an analogous structure (one-toone mapping and parallel connectivity), but it cannot cope well with inconsistent, complex structures which likely occur when concepts are compared.

The main problem with transformational models is the lack of a suitably generic set of transformations for geo-spatial concepts. Therefore, this similarity measure is not yet applicable in practice.

\section{Representing Semantics in the Hybrid Similarity Measure}

The hybrid similarity measure presented in this paper overcomes the mentioned shortcomings by combining a geometric model using Gärdenfors' conceptual spaces with a network model of (primarily spatial) relations. It supports two representational elements: properties modeled on dimensions and relations between concepts $^{2}$.

(a) Conceptual Space

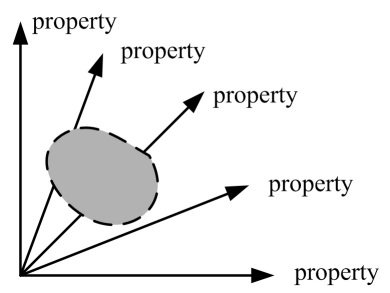

(b) Semantic Net

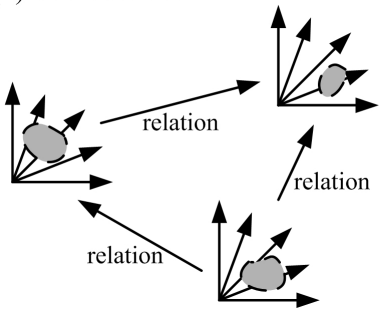

Figure 5: The hybrid similarity measure combines conceptual spaces with semantic networks.

First, each concept is described by its properties within a conceptual space. Second, concepts are related via links in a network structure to other concepts which are again described in conceptual spaces. Figure 5(a) describes a conceptual space and (b) the semantic network structure.

${ }^{2}$ Strictly speaking, relations do not hold between concepts, but between instances of these concepts. 


\subsection{Representing Properties}

Properties describe qualities or traits of individuals belonging to a concept. Gärdenfors (2000) proposed conceptual spaces as a cognitively plausible framework for representing information at a conceptual level. A conceptual space is a multidimensional space built from domains. Concepts are represented via multidimensional regions (so-called conceptual regions) within a conceptual space, describing a concept through its properties on quality dimensions in the domains.

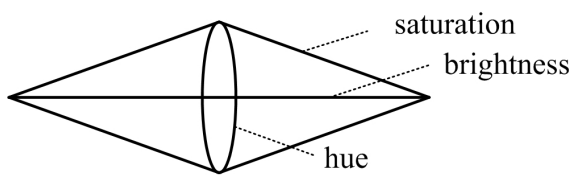

Figure 6: The color spindle with its dimensions hue, saturation and brightness.

A domain is built from one or several integral quality dimensions, which are not separable by the human perception. The color and the space domains may serve as introductory examples: humans perceive color as an integral phenomenon being composed of three quality dimensions hue, saturation and brightness. The space domain can be modeled as a 3-dimensional Euclidean space built of the dimensions height, width and depth. It is impossible to perceive the height of something physical without its width or depth. Besides those domains which are based on quality dimensions perceived by our sensory receptors, abstract, nonsensory dimensions can be posited. These dimensions play a major role in the semantic description of scientific concepts, as in our running example.

The quality dimensions, but also the domains themselves, may be correlated or functionally dependent. Such dependencies result in non-orthogonal dimensions of the conceptual space. For example, in the color domain, the dimensions saturation and brightness are coupled. When brightness reaches its extreme points of black or white, the human eye perceives no saturation of colors.

Quality dimension. A quality is the basic entity which humans can perceive or measure (Masolo, Borgo, Gangemi, Guarino, \& Oltramari, 2003). Shape, color and size are qualities perceived by our sensory system while qualities such as radiation or the time period of being waterlogged are measured with the aid of some device. All qualities are modeled as quality dimensions (figure 7), which correspond to the way humans perceive similarity between stimuli.

The measured scale of a dimension has to be ordinal, interval or ratio (figure 8). In many cases the same dimension can be measured with different scales, e.g. waterlogged can be represented on an ordinal scale, but it can also be translated on a ratio scale by specifying the number of days per year. While an ordinal scale is not as exact as a ratio scale, it is easier to handle for users of a retrieval system. On the other hand, ordinal values can hide semantics in labels. The meaning of 


\section{SCHWERING, KUHN}

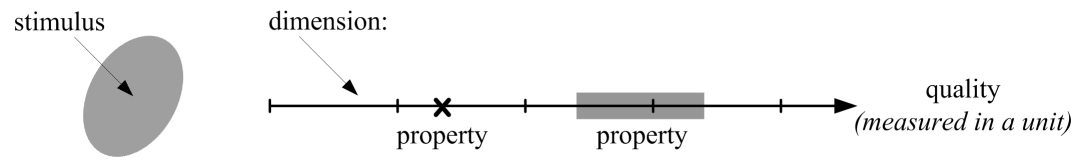

Figure 7: The weight of the stimulus is perceived by our sensory system. The quality weight is modeled as a dimension. Properties such as "heavy" are represented as a point or an interval on the quality dimension.

terms like seasonally, periodically and sometimes waterlogged may differ across information communities.

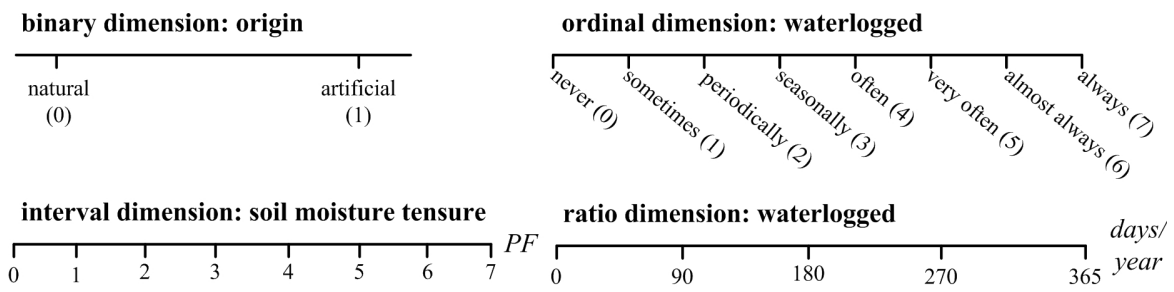

Figure 8: Qualities represented on dimensions with different scales.

Similarity and difference judgements generate an ordering on stimuli. Quality dimensions with different geometry or topology account for different orderings. Figure 9 (a-d) illustrates dimensions with different geometries to specify time periods: a time period can be represented by an interval on a time line (a), which is isomorphic to the line of real numbers. It may also be specified by its length: the half-line in (b) shows the numbers of days waterlogged and the line with two end points 0 and 365 represent the days per year a floodplain is waterlogged (c). For particular purposes, it makes sense to model time as a circular dimension: the flooding period of a floodplain is described on a circular dimension of months of a year (d) to indicate that the distance between November and December is the same as between January and December. Figure 9 (e) is an example for a discrete geometric structure. Land covers cannot be ordered linearly according to their similarity, but are grouped into different categories. The choice of the geometry has a fundamental influence on the measured similarity and must be done individually for every retrieval task.

\subsection{Representing Relations}

Relations describe the connection between two concepts, between a concept and its property or between two properties (figure 10). The two latter relation types are 
A HYBRID SIMILARITY MEASURE 13

(a)

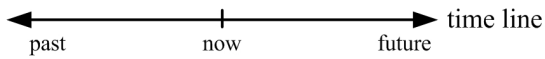

(b)

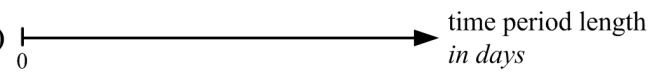

(c)

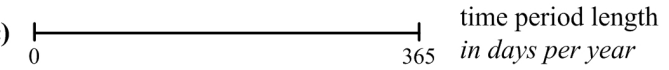

(e) surface

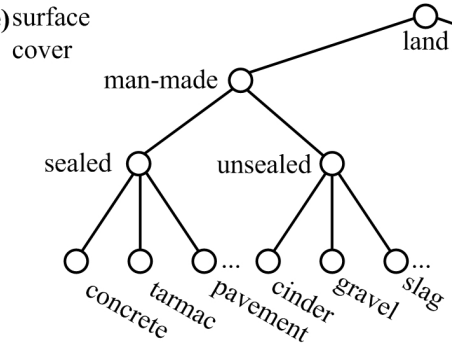

in days per year

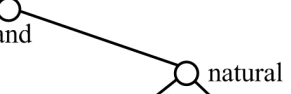

(d) time period

in months of a year

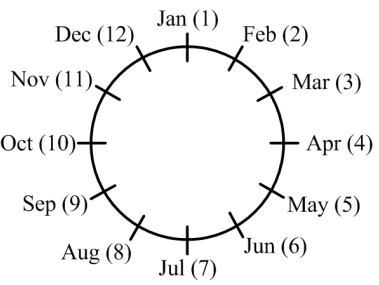

Figure 9: Geometry and topology of dimensions depend on the similarity perception.

partly supported by the structure of conceptual spaces. The relation "the concept floodplain has the property sometimes waterlogged" is represented by a conceptual region occupying a specific range on the quality dimension. The relation between two properties belonging to the same quality is modeled by the relation of the intervals (representing the properties) along a dimension. The distance between two intervals represents their similarity.

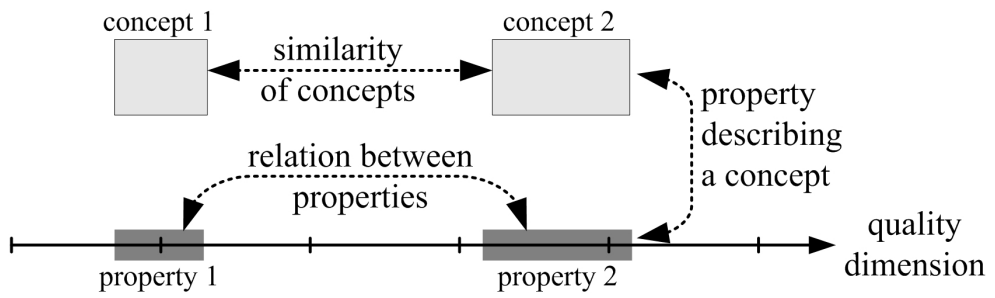

Figure 10: Concept-property and property-property relations are modeled inherently in a conceptual space. Concept-concept relations are limited to similarity; semantic relations such as "next to" are not possible to represent.

The semantic relation between two concepts is not representable within a single conceptual space. Two concepts described in the same conceptual spaces have only a spatial relation, which represents the semantic distance, i.e. the similarity. To account for semantic relations between concepts, the representational model 


\section{SCHWERING, KUHN}

of the hybrid similarity measure combines conceptual spaces with semantic networks. Each node of the semantic network represents a concept, which is related via links to other concept nodes in the net. The concept themselves are described within a conceptual space (figure 5).

In the hybrid similarity measure, relations between concepts either hold or do not hold, but they cannot have any degree of existence. Like properties, different types of relations exist: we distinguish between hierarchic and partonomic relations, which are commonly used in ontologies, as well as association relations, such as spatial relations. If the order of arguments is relevant to the meaning of the relation, the user of the semantic information retrieval system must specify the direction of the relation. In contrast to most similarity algorithms using network models, the hybrid similarity measure only matches relations with the same orientation. The following list shows how the different types of relations can be used for structured representation and to define the scope of properties:

1. The property refers to the whole concept. This property is represented as one domain of the conceptual space. No relational structure is needed.

2. Is-a relation: the property refers to a superconcept. In this case, the superconcept and the concept are modeled as two separate concepts with two conceptual spaces related via an is-a relation. For computing the similarity, a concept inherits the additional properties of superconcepts.

3. Part-of relation: the property refers to a part of the concept. In this case, the concept and its part are modeled as separate concepts related via part-of relation.

4. Non-taxonomic relation: the property refers to a specific aspect, e.g. it describes the spatial environment where instances of a concept are usually located. In this case, the spatial environment is modeled as a separate concept (e.g. river valley or river) and related to the original concept via a non-taxonomic relation (e.g. contained within or next to). With this non-taxonomic relation any scope can be represented explicitly.

In the first two cases, the domains are part of the original concept. In the third and fourth case, the hybrid similarity measure includes this information by comparing the similarity of the related concepts.

In analogy to the property specification of concepts, the specification of relations starts with selecting the relevant relations and the concepts the relation refers to. After specifying the direction, weights can be chosen to determine the importance of the relation. Then, the related concept needs to be described within its conceptual space. The related concept again may have relations to other concepts. This iterative process stops when the user of an information retrieval system decides that further descriptions do not contribute significantly to specify the query. 


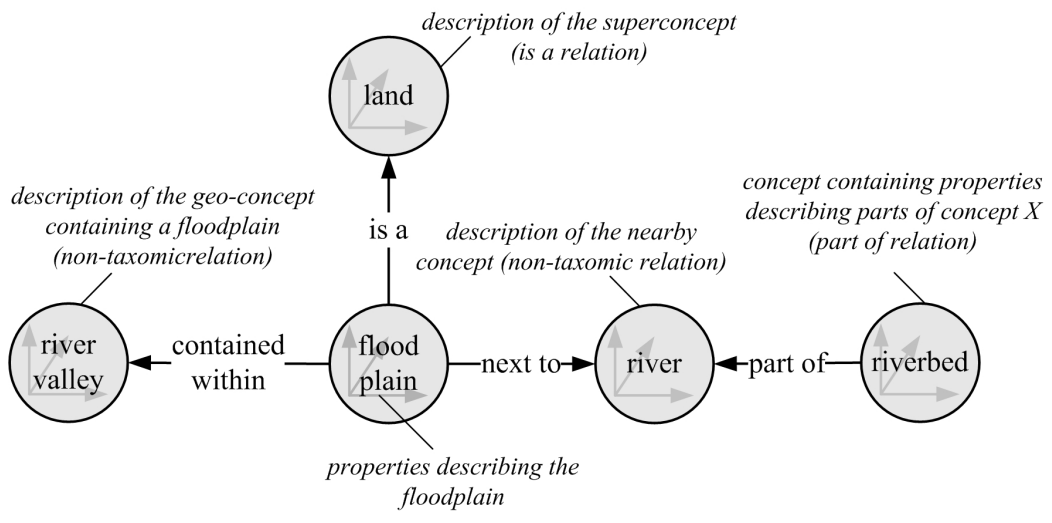

Figure 11: The structured representation of the semantics of a floodplain.

Like the similarity measure of Rodriguez and Egenhofer we call all concepts, directly or indirectly related to a concept $C$, the semantic neighborhood of $C$. A user specifying a query concept decides on the size of a semantic neighborhood for the retrieval.

\subsection{Specific Requirements of Information Retrieval}

Concepts are described in their own conceptual space built from a selection of relevant domains and relations. However, the similarity measure between a query concept and the concepts in a data source requires a common space. Since similarity for information retrieval is a directed similarity, the only concept which determines the space for the retrieval is the query concept. Only those qualities and relations, which are used for the query concept specification, influence the similarity. Additional qualities or relations from concepts in the data source are ignored, because they are not relevant for the query. Not all data source concepts are described by all query concept qualities or relations. This absence influences the similarity negatively. Only if a matching quality or relation is found, their values are compared. The selection of domains and their dimensions depend on the purpose of the semantic description and the expertise of the person creating the specification. While the ecosystem around a river is important for the specification of a river from an ecologic perspective, its relation to the riverbank plays a minor role in a navigation context. The representational model of the hybrid similarity measure considers context by the choice of domains and their importance. The user of a semantic information retrieval system can represent the context and significance of dimensions via assigning different weights to domains and dimensions. Usually it is sufficient to limit the choice of three different levels, e.g. 


\section{SCHWERING, KUHN}

defining property or relation, characteristic property or relation and not important property or relation.

Each quality dimension has a scale and a geometry. Here again the query concept determines the common space, because similarity shall be measured from the user's point of view, within the user's cognitive space. The scale and the geometry of dimensions have an influence on the similarity measure. Figure 12 (a) shows two dimensions of waterlogged with an ordinal and a ratio scale and illustrates how one could be mapped on the other. Figure 12 (b) shows two dimensions with different geometry: depending on the context, the months of the year are modeled on a linear or on a circular dimension. This has an influenced on the distances measured on the dimensions and therefore on the similarity: A linear dimension expresses that January is more similar to the months of the year's beginning than to those of its end, while the circular dimension reflects the restart of the year after December. The user has to choose the geometry applicable in his context. The geometry and topology of dimensions are central to similarity measurement; the specification of the semantics can be done independently though.

(a) transformation: ordinal scale $\rightarrow$ interval / ratio scale

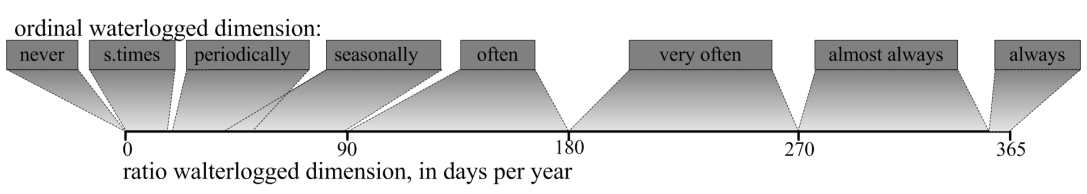

(b) transformation: linear geometry $\rightarrow$ circular geometry
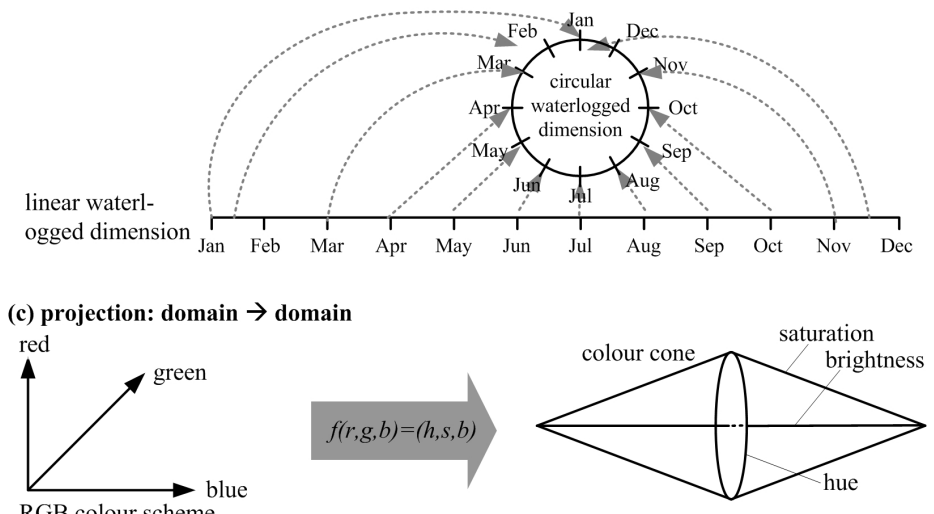

Figure 12: Dimensions with different scale (a) or geometry (b) are transformed into the quality dimension which represents the user's similarity perception. The same quality color is represented with different color schemes (c). The similarity is measured within the cognitively most suitable conceptual space of the user. 
How qualities are modeled in a conceptual space depends on the conceptualization of the world. For example, the quality color can be represented by different color schemes (Falk, Brill, \& Stork, 1986; Wyszecki \& Stiles, 2000). Color is distinguished in a spectrum by the wavelength and by the absorption. Monitors work with the additive color scheme RGB, while subtractive mixture of colors such as CMYK is common in the print media. The color spindle based on the dimensions hue, saturation and brightness reflects the human similarity perception of colors and the spatial distances reflect human similarity perception. The purpose of our approach to information retrieval is to provide users with data, which they consider to be similar to what they are looking for. Therefore, the retrieval system shall choose that color scheme for similarity measure which best represents the user's similarity perception. In case of a human user the color cone is the cognitively adequate model for the similarity measure. If the user is a machine, i.e. a web-service looking for the best-matching input, the information retrieval system uses a color scheme best matching the requirements of the web service.

\section{Semantic Similarity in the Hybrid Similarity Measure}

The proposed hybrid similarity measure is based on the notion of similarity of geometric models. Conceptual spaces provide a natural way of representing similarities: the spatial distance between two concept representations is interpreted as their semantic distance. The hybrid similarity measure addresses two problems of geometric similarity measures: firstly current approaches confine themselves to estimating the similarity between individuals, i.e. between points in the vector space. Concepts, however, are described by regions. The hybrid similarity measure reduces the n-dimensional problem to an one-dimensional one and computes the semantic distance between the intervals describing the query concept and the compared concept on each dimension. Secondly, since conceptual spaces are limited to properties, traditional geometric similarity measures do not include relations. The hybrid similarity measure however, accounts for relations via including the similarity of related concepts in the overall similarity measure.

\subsection{Dimension-Wise Comparison of Concepts}

The hybrid similarity measure shall determine the semantic distance between conceptual regions. Other similarity measures have solved this problem by reducing the regions to a single instance, e.g. to the nearest points of both regions or to the prototype, where the prototypical instance of each concept is assumed to be the central point or the centroid of each concept. Alternatively, a concept can be described by a set of exemplar instances. The average semantic distance between the exemplars is taken as semantic distance between both concepts. However, reducing the conceptual region to a single point results in information loss. More- 


\section{SCHWERING, KUHN}

over, the exemplar approach requires an extensional concept definition. Since the user of an information retrieval system specifies the query concept intensionally, there are no instances of the query concept available. It is necessary to include the whole conceptual regions in the distance calculation to draw valuable conclusions on the similarity of these concepts.
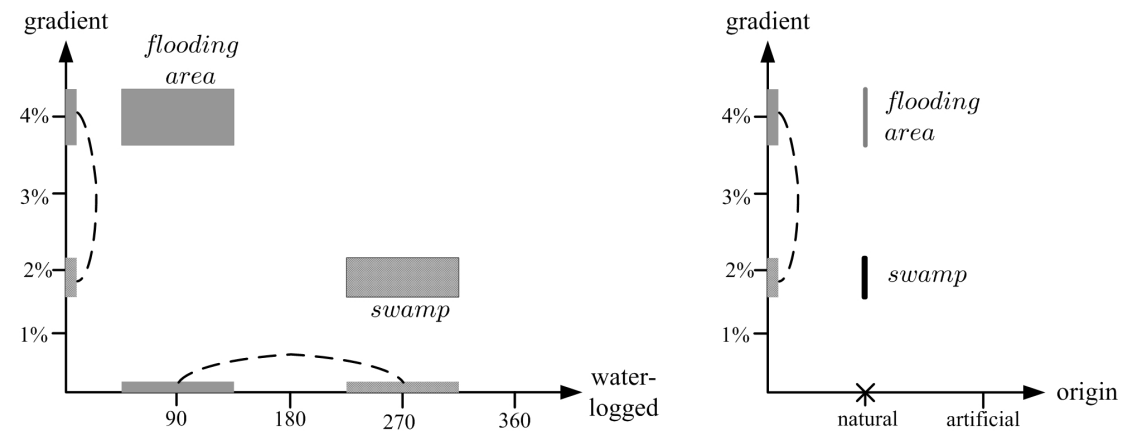

Figure 13: Conceptual regions are specified via points or intervals on dimensions.

The hybrid similarity measure reduces the $\mathrm{n}$-dimensional measurement problem to one-dimensional ones. Computing dimension-wise distances makes it possible to use the Minkowski metrics for similarity calculation. The Minkowski metrics (equation 1) estimate the spatial distance between two points by composing their dimension-wise distances. The city-block metric simply sums up the dimension-wise distance; the Euclidean metric sums up the squared distances and extracts its root. As illustrated in figure 13 concepts occupy either an interval or a point on each quality dimension. The distance between two points on a dimension is simply their separation. The distance between two intervals on a dimension is calculated accounting for the length of the intervals, the length of the overlap and the distance between the intervals, if they are disjoint. For semantic information retrieval, however, the distance calculation must be extended to account for the particular requirements of a retrieval task. In the following, we structure the search criteria in five types according to how similarity is defined (figure 14).

The first criterion type refers to points. Maximum similarity is reached when an individual is described by exactly the same value on the dimension. The more the values differ, i.e. the higher the distance computed via Minkowski metrics is, the lower is the similarity (figure 15). This criterion type applies in our case study, if the user searches for the concept river, which she describes with the property 365 days waterlogged a year. A water body which contains water almost all the year is more similar to the query than a water body which regularly dries up.

The second criterion type refers to exact intervals. Maximum similarity is reached when a concept agrees on the exact interval with the query. Any deviation 
A HYBRID SIMILARITY MEASURE 19

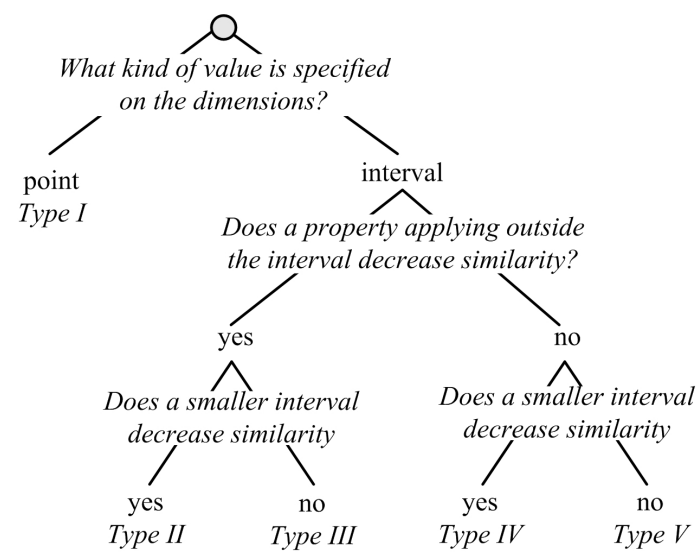

Figure 14: The decision tree to specify the notion of similarity for properties.

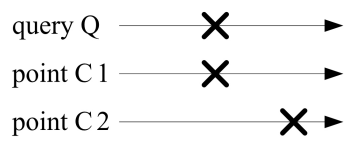

Figure 15: Properties of type I "point".

reduces the similarity. This criterion applies, for example, if the user searches for the concept flooding area which she describes with the property being waterlogged for between 60 and 90 days a year.

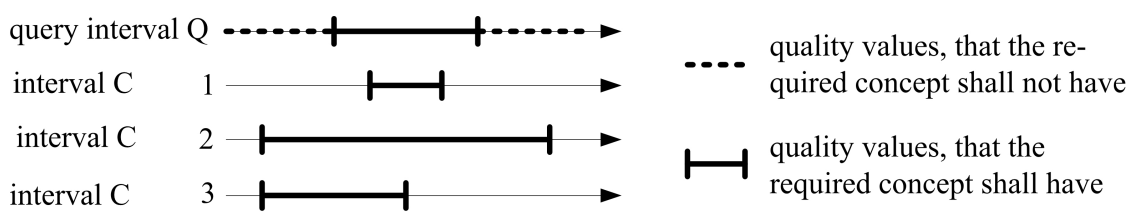

Figure 16: Properties of type II "“exact interval" and type III "maximum interval".

Figure 16 illustrates the query concept and the different match situations. In situation 1, the interval describing concept $C$ is smaller than the query interval; in situation 2 it is larger and in situation 3 it partially overlaps the query interval. When the user specifies the values of this dimension, she wants to say that the concept she searches for is neither less nor more days waterlogged a year.

A formula to compute the spatial distance between intervals with the underlying similarity notion of criterion type II is presented in equation 4 (see appendix for the definition of the interval operations). If interval $C$ is exactly the same as $Q$, 


\section{SCHWERING, KUHN}

the distance evaluates to zero. If $C$ is smaller than $Q$, the length of the overlap is smaller and the resulting distance is the length of the non-overlapping parts of $Q$. If $C$ is greater, $d_{d i m V a l}$ is the length of the non-overlapping part of $C$. If $C$ overlaps $Q, d_{d i m V a l}$ is the sum of the non-overlapping parts of $Q$ and the nonoverlapping parts of $C$. If $C$ and $Q$ do not overlap at all, $d_{\text {dim } V a l}$ is the length of the subtraction of $Q$ and $C$.

$$
d_{\text {dimVal }}(Q, C)= \begin{cases}l(Q)-l(Q \cap C)+l(C \mid Q) & \text { if }(Q \cap C) \neq \emptyset \\ l(Q-C) & \text { if }(Q \cap C)=\emptyset\end{cases}
$$

The third criterion type refers to maximum intervals. Maximum similarity is reached when a concept has the same or a smaller interval. For example, the user specified a range for the gradient that the concept flooding area should maximally vary in. Since not all concepts necessarily are described by such a great variation regarding the gradient, it is enough that concepts fulfill only a subsection of the query interval. Therefore, also situation 1 in figure 16 would lead to maximum similarity.

The similarity function proposed in equation 5 is adequate for this criterion type. If $C$ is greater than $Q, d_{\text {dim } V a l}$ is the length of the non-overlapping part of $C$. If $C$ is exactly the same or smaller than $Q, d_{\operatorname{dim} V a l}$ is zero. If $C$ and $Q$ do not overlap, $d_{\operatorname{dimVal}}$ is the length of the subtraction of $Q$ and $C$ minus the length of $Q$, because the nearest point in $Q$ is taken as reference point.

$$
d_{d i m V a l}(Q, C)= \begin{cases}l(C)-l(Q \cap C) & \text { if }(Q \cap C) \neq \emptyset \\ l(Q-C)-l(Q) & \text { if }(Q \cap C)=\emptyset\end{cases}
$$

The fourth criterion type refers to minimum intervals. Maximum similarity is reached when a concept covers at least the same interval as the query. Only smaller intervals have a negative impact. For example, the user searches for the flooding areas which are flooded during autumn, but she does not care whether the flooding period is longer or occurs twice a year.

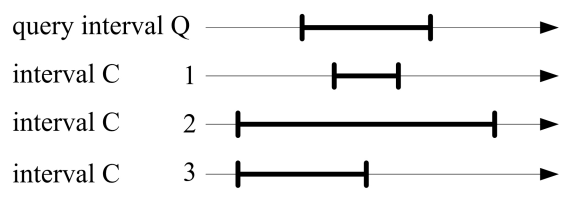

Figure 17: Properties of type IV "minimum interval" and type V "one of interval".

Figure 17 illustrates the query interval and the different match types: when the user specifies the values for this dimension, she does not say anything about 
A HYBRID SIMILARITY MEASURE 21

\begin{tabular}{|l|l|l|l|l|l|}
\hline Type of match & point & $\begin{array}{l}\text { exact } \\
\text { interval }\end{array}$ & $\begin{array}{l}\text { maximum } \\
\text { interval }\end{array}$ & $\begin{array}{l}\text { minimum } \\
\text { interval }\end{array}$ & $\begin{array}{l}\text { one of } \\
\text { interval }\end{array}$ \\
\hline Exact match & ok & ok & ok & ok & ok \\
Smaller interval & - & neg. & ok & neg. & ok \\
Larger interval & - & neg. & neg. & ok & ok \\
Overlapping interval & - & neg. & neg. & neg. & ok \\
No overlap & neg. & neg. & neg. & neg. & neg. \\
\hline
\end{tabular}

Table 1: Various matches influence similarity differently depending on the criterion type: "ok" indicates that the interval satisfies the query; "neg." indicates that the interval does not completely satisfy the query.

concepts for which values outside the interval also apply. Therefore, situation 2 leads to maximum similarity.

Equation 6 formalizes the matching of the fourth criterion type: The distance is zero, if $C$ and $Q$ are the same or if $C$ is greater than $Q$. Only in the case where $C$ is not overlapping completely $Q$, the distance is greater zero. If $C$ and $Q$ do not overlap, the distance is computed as the length of the subtraction of $C$ and $Q$ minus the length of $C$, because the additional values of $C$ do not influence the similarity.

$$
d_{\text {dimVal }}(Q, C)= \begin{cases}l(Q)-l(Q \cap C) & \text { if }(Q \cap C) \neq \emptyset \\ l(Q-C)-l(C) & \text { if }(Q \cap C)=\emptyset\end{cases}
$$

The fifth criterion type refers to intervals, where maximum similarity is reached when a concept is described by at least one value that is part of the query interval (one of interval). Only non-overlapping intervals affect similarity negatively. For example, the user describes the query concept flooding area additionally by the quality fertility. In her conceptualization, a flooding area is fertile or even very fertile. The similarity notion "one of interval" means that the user accepts any concept, that is described by a fertility degree fertile or very fertile and she does not care whether other fertility degrees apply as well. Situation 1 to 3 (figure 17) all have $d_{\operatorname{dim} V a l}=0$ to the query interval. Equation 7 computes the distance according to the notion of similarity of type V. The distance of two non-overlapping concepts is measured by the length of the subtraction of $Q$ and $C$ minus the length of both intervals, because the nearest points in $C$ and $Q$ are taken as reference points.

$$
d_{d i m V a l}(Q, C)= \begin{cases}0 & \text { if }(Q \cap C) \neq \emptyset \\ l(Q-C)-l(Q)-l(C) & \text { if }(Q \cap C)=\emptyset\end{cases}
$$

To measure the overall spatial distance between concepts $c$ and $q$ the distance of each property is summed up according to the slightly modified formula of the 


\section{SCHWERING, KUHN}

Minkowski metrics. $Q_{i}$ is the value on dimension $i$ of query concept $q$ and $C_{i}$ for concept $c$.

Table 1 summarizes all different matching types and gives an overview of interval relations and their influence on similarity.

Standardisation of Properties. The fact that vector spaces have a metric allows for the calculation of distances between points or intervals in the space. In order to calculate these semantic distances between individuals and concepts it is required that all quality dimensions are represented in the same relative unit of measurement. Two standardizations have been investigated in this retrieval context: percent ranks and z-transformation.

Percent ranks calculate how many concepts in the data source have a lower or higher value on the respective quality dimension. To compute percent ranks the quality dimensions are divided into intervals. All values within one interval fall into one category. The frequency of each category is computed by the number of occurrences over the data source. The similarity measure uses the cumulative frequencies for the calculations instead of the actual values. The cumulative frequency distribution is the successively summed up category frequencies (equation 8): percentrank $k_{k}$ is the cumulative percent value for the category $k, f_{\text {cum }}(c)$ is the cumulative frequency of the category $c$ and $n$ is the size of the collective.

$$
\text { percentrank }_{\% k}=\frac{f_{\text {cum }}(c)}{n} * 100 \%
$$

The standardization with percent ranks takes only the order of values into consideration. The actual values do not influence the percent ranks. Therefore, percent ranks are applicable for ordinal dimensions only (Fahrmeir, 2004).

The z-transformation standardizes values of a quality dimension such that the mean value is zero and the statistical dispersion is one. It compares the deviation of values to the mean value: a $\mathrm{z}$-value divides the deviation of a value to the mean value by the size of the collective. Equation 9 shows the formula to compute the $\mathrm{z}$-value $z_{i}: x_{i}$ is the original value on the quality dimension, $\bar{x}$ is the mean value (typically the arithmetic mean value) and $s$ is the dispersion (typically the standard deviation). As the z-transformation is also applicable to interval- or ratioscaled values (Devore \& Peck, 2001; Fahrmeir, 2004), we used it in our evaluation (section 6.

$$
z_{i}=\frac{x_{i}-\bar{x}}{s}
$$

Both standardizations - the percent rank and the z-transformation - standardize single values. Thus, the hybrid similarity works with intervals on each dimension. Assuming that every interval has a symmetric distribution - typically a uniform or normal distribution - we can simply use the mean value of each interval for the standardizations. To standardize skew intervals, e.g. gamma distributed values, we need to include the distribution in the calculations. 
Matching conceptual spaces with different quality dimensions. A common problem of all (geometric) similarity measures discussed previously is their inability to deal with concept descriptions which are based on different quality dimensions. The qualities used to describe the query concept are not always applicable to data sources. In the case study, we describe water bodies by their gauge height. Concepts which cannot be described by the quality gauge height have no common multidimensional space with water bodies and therefore the semantic distance cannot be computed.

$$
\text { semdist }_{d i m}=\alpha * d_{\text {dimExist }}\left(\operatorname{dim}_{q}, \operatorname{dim}_{c}\right)+\beta * d_{d i m V a l}(Q, C)
$$

The hybrid similarity therefore includes the existence of quality dimensions and the dimensional semantic distance in the similarity measure. Equation 10 shows the formula: $d_{\operatorname{dim} E x i s t}\left(\operatorname{dim}_{q}, \operatorname{dim}_{c}\right)$ returns 0 if the dimensions $\operatorname{dim}_{q}$ and $\operatorname{dim}_{c}$ match and a value greater 0 if they do not match (the exact value of $d_{\text {dimExit }}$ depends on the standardization). Dimensions match and mismatch. There is no graded similarity. The second term, $d_{\operatorname{dim} V a l}(Q, C)$, compares the intervals on the respective dimension according to equations 4-7. We chose a ratio of $\alpha=0.8$ for the dimension existence $d_{\text {dimExit }}$ and $\beta=0.2$ for the dimensional semantic distance $d_{d i m V a l}$. We empirically tested different ratios in our evaluation and found the best results for these values (Schwering, 2007b).

\subsection{Including Related Concepts in the Similarity Measure}

Besides properties, the hybrid similarity measure shall include also relations to other concepts modeled within the semantic network. The complete procedure comprises three steps: step one is the alignment of matching representational elements and the calculation of their semantic distances on quality dimensions of the conceptual space (figure 18). Step two and three deal with the integration of relations and related concepts in the overall similarity measure (figure 19).

The steps can be described as follows:

1. Identify all common and corresponding quality dimensions of query concept $q$ and the compared concept $c$, as well the dimensions of concepts related to $c$ and $q$. Step 1.a in figure 18 illustrates the alignment of matching dimensions in the conceptual space of $c$ and $q$. Inherited dimensions are treated as ordinary dimensions of a conceptual space. Steps 1.b.1 and 1.b.2 show the alignment of related concepts. Spatial relations are compared semantically: either this comparison is based on the relation names, or it uses a special framework to determine the semantic similarity of spatial relation as proposed in (Li \& Fonseca, 2006) or (Schwering, 2007a). In the example, there are no correspondences for the quality dimensions D5 and D6 and for 


\section{SCHWERING, KUHN}

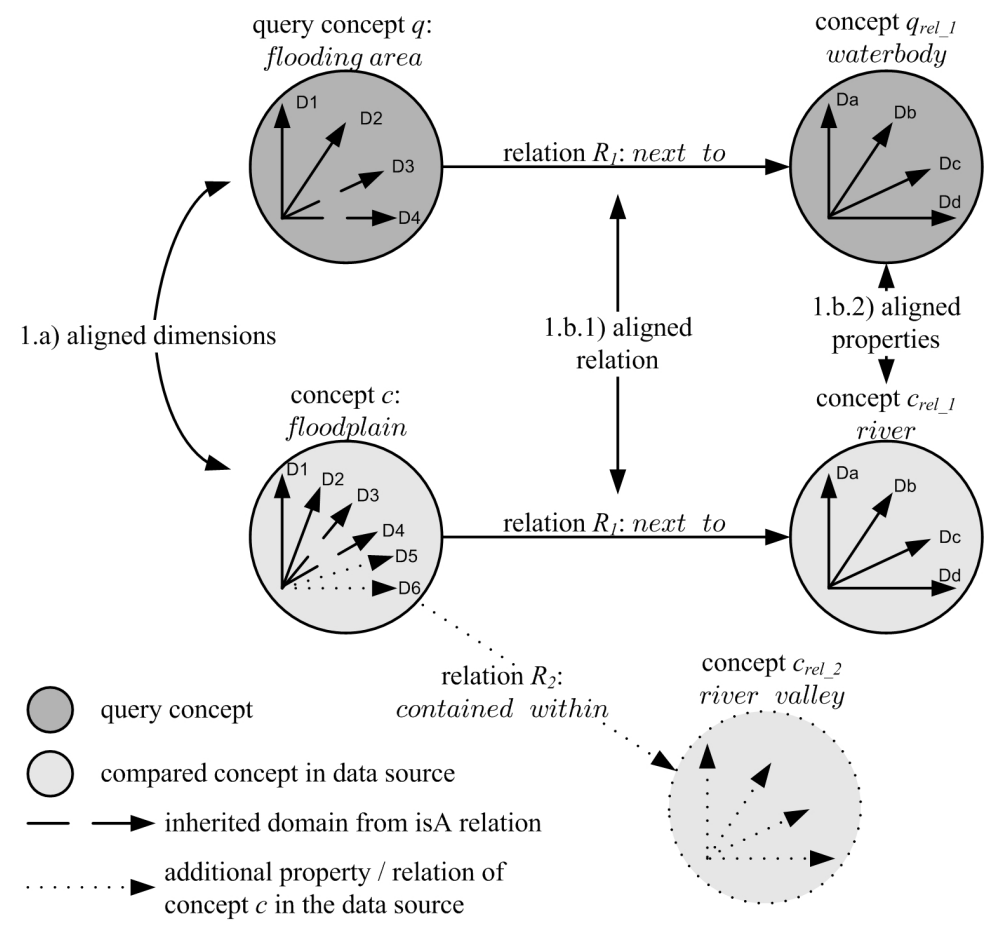

Figure 18: Step 1 comprises the semantic distance calculation on dimensions of the conceptual space and inherited quality dimensions.

the relation 2. All additional properties and relations of concept $c$ that the query concept $q$ does not contain are ignored in the similarity measurement.

2. For each related concept $c_{r e l}$ and $q_{r e l}$ compute a semantic distance value analogous to the similarity measure of conceptual spaces, e.g. a semantic distance value for the concept waterbody to river both being related via a next to relations. The resulting distance value is the Euclidean distance between water body and river (step 2.a in figure 19).

3. Compute the difference between $c$ and $q$ for each domain belonging to their conceptual space (step 3 in figure 19).

The result is an $\mathrm{n}$-dimensional vector (equation 11); $n$ is the sum of the common dimensions of the conceptual space of $c$ and $q$ plus the dimensions for its related concepts - in this example $n=5$ : four domains of $c$ and $q$ and one related for the related concept. Each dimension of this vector represents the dimension-wise distance: 


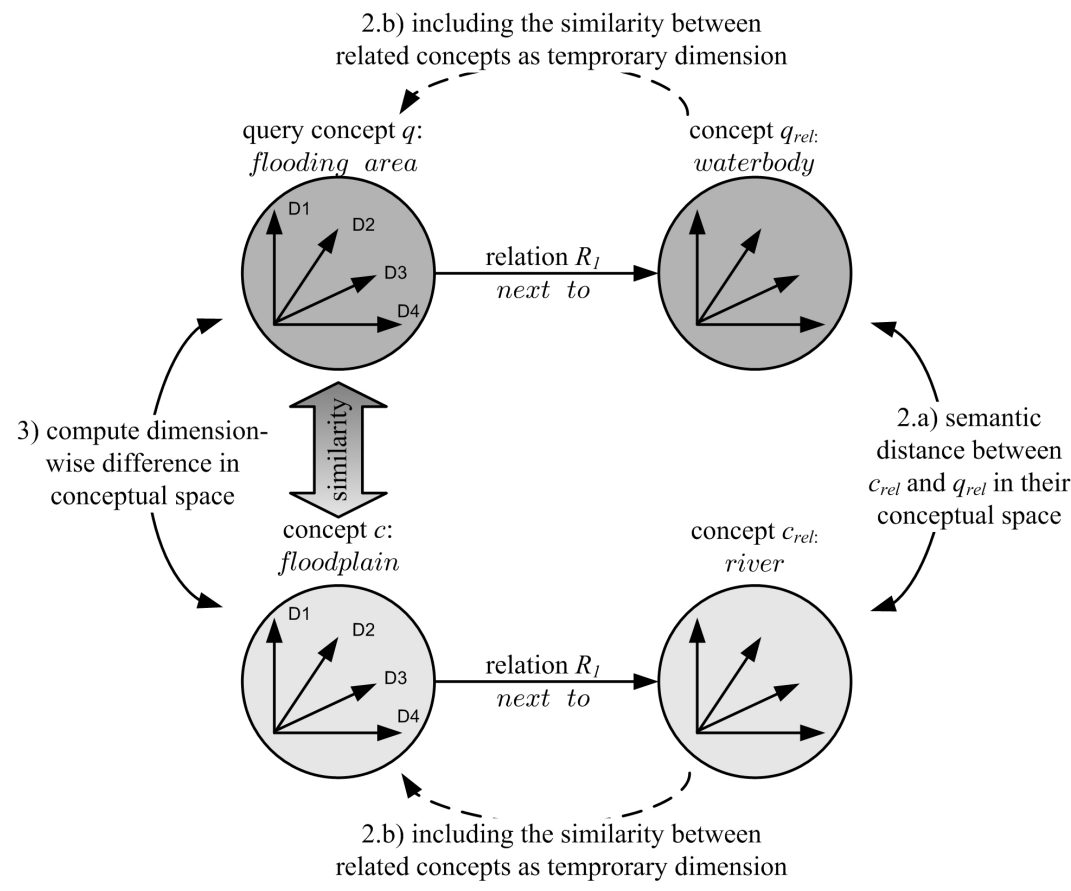

Figure 19: Steps 2 and 3 measuring the similarity between query concept $\mathrm{Q}$ (flooding area) and the data source concept C (floodplain).

- either it is the difference between the concept's value on this dimension and the query concept's value on this dimension

- or it is the Euclidean distance between the related concept $c_{r e l}$ and the related concept $q_{r e l}$.

$$
\left(\begin{array}{l}
c_{D 1}-q_{D 1} \\
c_{D 2}-q_{D 2} \\
c_{D 3}-q_{D 3} \\
c_{D 4}-q_{D 4} \\
c_{r e l}-q_{r e l}
\end{array}\right) \quad\left\{\begin{array}{l}
\text { dimension-wise distance } \\
\text { semantic distance between related concepts }
\end{array}\right.
$$

Analogous to the semantic distance on dimensions (equation 10) we include the existence of the relation in the semantic distance. Equation 12 shows the corresponding formula for relational semantic distance.

$$
\operatorname{sem~dist~}_{\text {rel }}=\alpha * d_{\text {relExist }}\left(r e l_{q}, \operatorname{rel}_{c}\right)+\beta * d_{\text {relVal }}(q, c)
$$




\section{SCHWERING, KUHN}

These semantic distance values are fed into either the Euclidean or the cityblock distance formula. In the query concept, the user may weigh each quality dimension by its importance. These weighting factors are included in the similarity measure by a dimension-wise multiplication factor $w$ (Raubal, 2004):

$$
\text { sem dist }_{\text {concept }}=\left(\sum_{i} w_{i} * \mid \text { semdist }\left._{\text {dim }_{i}}\right|^{r}+\sum_{j} w_{j} * \mid \text { semdist }\left._{r e l_{j}}\right|^{r}\right)^{1 / r}
$$

Including related concepts in the similarity measurement is an iterative process. To stop this process the user needs to decide on a neighborhood size.

\subsection{Algorithm of the Hybrid Similarity Measure}

Figure 20 gives an overview of the process to calculate the semantic distance between two concepts. It iterates through all dimensions and relations of the query concept. In case of a dimension, it searches for a matching dimension in the compared concept. If no matching dimension exists, it computes a standardized distance value for the dimension existence. If the hybrid similarity measure detects a dimension match, it compares the values on the dimension: the formula for computing the standardized distance depends on the dimension's geometry, scale and the notion of similarity. Since the non-existence of a dimension has more negative impact on the similarity than different values on the dimension, we weigh the semantic distance of the dimension existence with $\alpha$, the semantic distance between the dimension values with $\beta$ and $\alpha>\beta$.

In case of a relation, the hybrid similarity measure tries to find a matching relation. Analogous to dimensions, a standardized distance value for the relation existence is computed. If the hybrid similarity measure detects a relation match, it compares both related concepts recursively: the concept related to the query concept is the new query concept. The number of recursions depends on the size of the semantic neighborhood: In our case study we account only for directly connected concepts. Therefore the relations of the related concepts are not included in the similarity measurement. We again weigh the relation existence with $\alpha$ and the semantic distance between related concepts with $\beta$. The hybrid similarity measure sums up the semantic distances of the representational elements via the Euclidean or the city block metric. 
A HYBRID SIMILARITY MEASURE 27

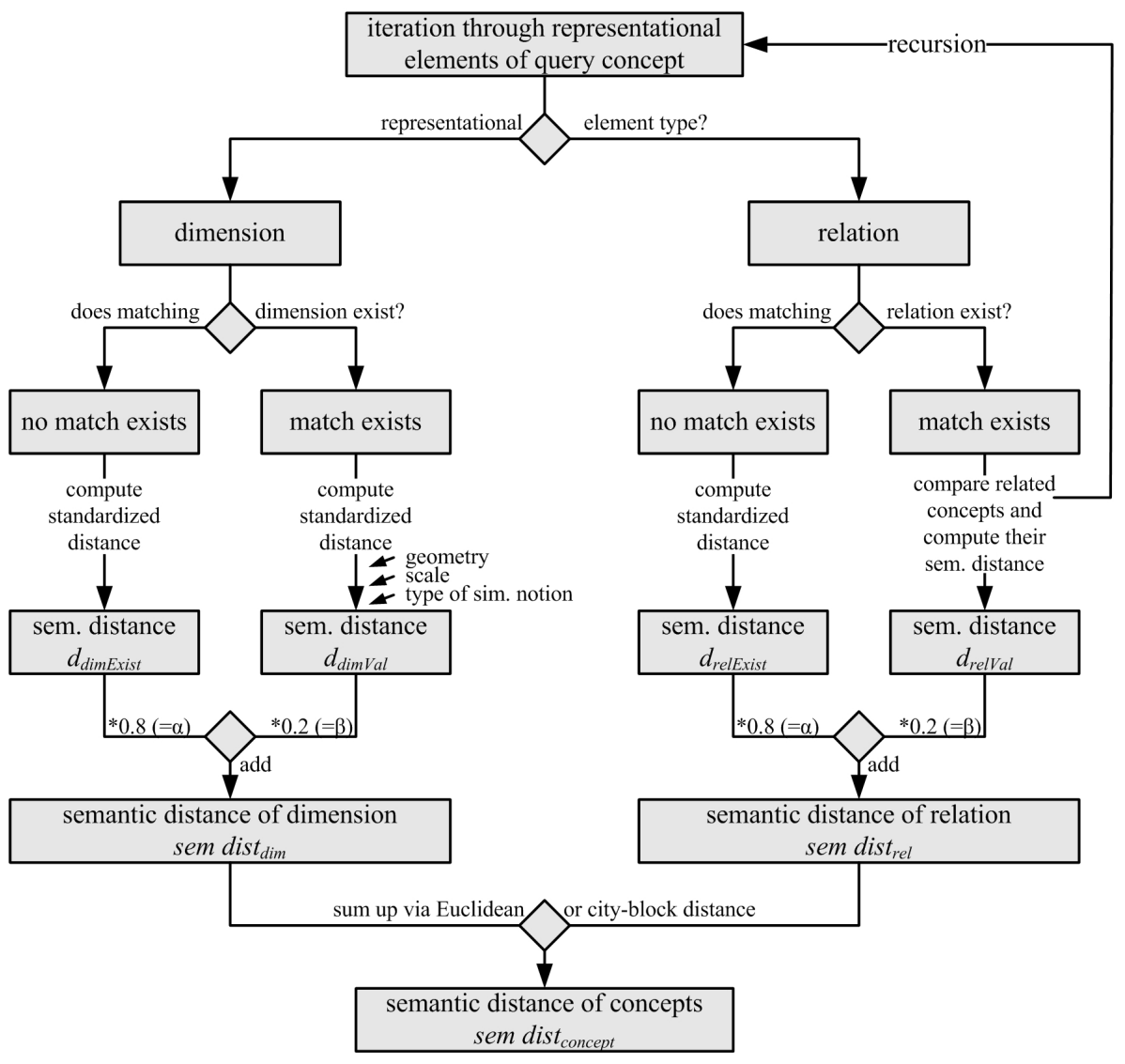

Figure 20: Overview of the hybrid similarity measure.

\section{Evaluation of the Hybrid Similarity Measure}

\subsection{Comparison of Similarity Measures}

We tested the effectiveness of our hybrid semantic similarity measure in our retrieval use case as follows: Experts ${ }^{3}$ created semantic descriptions of different data sets and query concepts. An effective similarity measure shall rank the concepts described in the data sets according to their relevance. We assume that those results which are most similar to the query concept are also the most relevant ones. In our study, an expert was asked to rank the concepts described in the data set

\footnotetext{
${ }^{3}$ The experts are GI professionals from Ordnance Survey and from the Institute of Geoinformatics of the University of Muenster, who are familiar with the data sets.
} 


\section{SCHWERING, KUHN}

according to their relevance. We compared the hybrid similarity measures against Gärdenfors conceptual spaces, the contrast and the ratio model as two representatives for feature models and Rada's DISTANCE as a network model. Alignment models could not be used because of their problem to represent complex and inconsistent semantics of concepts. Transformational models could not be applied, for lack of transformations.

In our case study, a user is searching for flooding areas, which are specified as low-lying, partially waterlogged land typically located next to a river. We investigated three different data sets (respectively specifications): Ordnance Survey's OS MasterMap, the German topographic-cartographic information system ATKIS (Amtliches Topographisch-Kartographisches Informationssystem) and the data transfer standard SDTS (Spatial Data Transfer Standard). From each data source we selected a comparable subset of concepts from the hydrology domain: land parcels - waterlogged and non-waterlogged - and water bodies. The set of partially waterlogged land parcels contains potentially relevant concepts for the query concept flooding area and the set of water bodies contain potentially relevant concepts for the related query concept river. Different experts had to set up semantic descriptions for the set of concepts of each data source using a predefined shared vocabulary. The shared vocabulary contained 22 different quality dimensions with their values, 7 spatial relations and is-a and part-of as hierarchic relations. The similarity measures were applied to the semantic descriptions created by the experts.

Figure 21 shows the results for the comparison in a normalized precision-recall curve in a retrieval task. A system's precision is its ability to retrieve only relevant information units and decline irrelevant ones. The recall value on the other hand expresses the number of retrieved relevant items out of all existing relevant information. Depending on the order of the retrieved documents, the development of recall and precision of retrieved documents is not continuous. To have a smooth development of precision and recall the curves are interpolated to a normalized precision-recall curve. The precision value of a concept must be at least as high as the precision values of the concepts ranked afterwards, so that the precision values are continuously decreasing (and analogous for recall). The higher the retrieval and the higher the recall (continuously over the curve), the more effective is the retrieval system.

For all data sources, the hybrid semantic similarity measure performs very well and in general it performs better than any of the other approaches. For the data set from OS MasterMap the conceptual spaces provide good results, too. The contrast and the ratio model reach only a precision of $0.80^{4}$, while DISTANCE reaches only 0.42 . For the data set from ATKIS, the hybrid similarity measure and conceptual spaces reach a precision of 1.00 , but conceptual spaces only a

\footnotetext{
${ }^{4}$ For visibility reasons we draw the curves with the same precision or recall values right beside each other in the figure.
} 

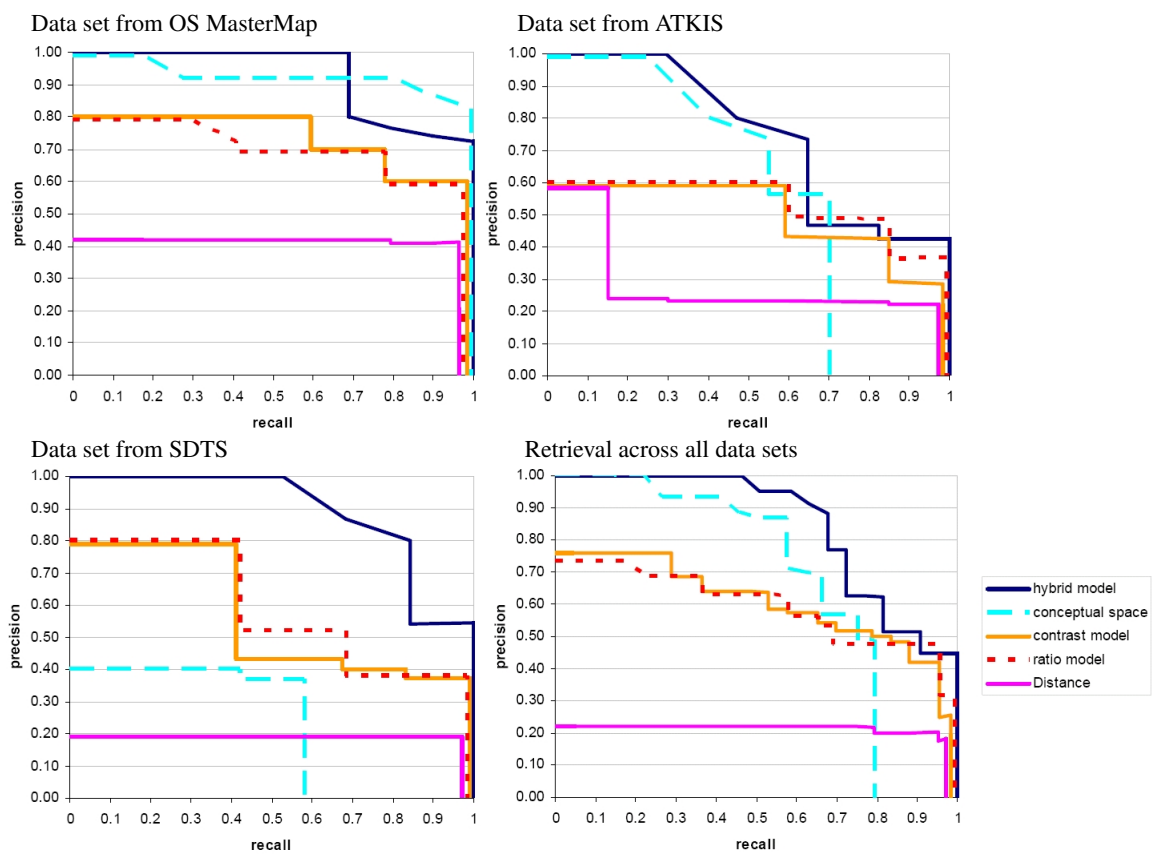

Figure 21: Comparison of Semantic Similarity Measures.

recall of 0.70 . The precision of the contrast model, ratio model and DISTANCE start with 0.60, but again the DISTANCE model performs worst. For the data set from SDTS, the hybrid similarity measure clearly returns the best results. Ratio and contrast model reach a precision of 0.80 . Conceptual spaces (max. precision 0.40 , max. recall 0.58) and DISTANCE (max. precision 0.19, max. recall 1.00) perform rather poorly. In the retrieval task across all data sets, the hybrid similarity measure performs best with a maximum precision and a maximum recall of 1.00. Conceptual spaces perform very well for the first hits (precision of 1.00), but reach only a recall of 0.79 . The contrast and the ratio model's precision are only 0.76 respectively 0.73 . DISTANCE performs worst with a maximum precision of only 0.22 . Table 2 shows how much the precision and recall values improve when using the hybrid semantic similarity measure: The clearest results are achieved when querying for flooding areas: The precision / recall are increased by $9.0 \% / 12.3 \%$ over the contrast model, by $8.6 \% / 12.1 \%$ over the ratio model, by $15.1 \% / 20.3 \%$ over the Conceptual Space and by $114.0 \% / 156.9$ over the DISTANCE approach (across all data sources). 
30 SCHWERING, KUHN

\begin{tabular}{|l|c|c|c|c|}
\hline & $\begin{array}{c}\text { Contrast Model } \\
\text { prec. / recall }\end{array}$ & $\begin{array}{c}\text { Ratio Model } \\
\text { prec. / recall }\end{array}$ & $\begin{array}{c}\text { Conceptual Space } \\
\text { prec. / recall }\end{array}$ & $\begin{array}{c}\text { DISTANCE } \\
\text { prec. / recall }\end{array}$ \\
\hline flooding area & $9.0 / 12.3$ & $8.6 / 12.1$ & $15.1 / 20.3$ & $114.0 / 156.9$ \\
river & $2.3 / 2.5$ & $0.4 / 0.5$ & $74.9 / 86.3$ & $16.1 / 54.3$ \\
\hline \hline average & $5.6 / 7.4$ & $4.5 / 6.3$ & $45.0 / 53.3$ & $65.1 / 105.6$ \\
\hline
\end{tabular}

Table 2: Improvement of the precision and recall values in $\%$ when applying the hybrid similarity measure.

\subsection{Discussion of the Results}

The results of the evaluation show a clear advantage of the hybrid similarity measure. We see one of the main reasons in the expressivity of the hybrid approach: While conceptual spaces, the contrast and the ratio model account only for properties and DISTANCE only for relations, the hybrid similarity measure can use a richer semantic description containing properties as well as relations, which leads to more accurate results. The hybrid similarity measure combines conceptual spaces to model properties and network models: conceptual spaces are preferred to feature sets, because they provide more structure by separating the quality from the quality value. The evaluation shows that in most of the queries conceptual spaces outperform the feature models (contrast and ratio). Since conceptual spaces require a common space, they often do not reach a recall of 1.00. To overcome this disadvantage, the hybrid similarity measure extends conceptual spaces to check the existence of qualities separately from the comparison of quality values.

A second difference to standard approaches is the way the hybrid similarity measure determines the similarity. Similarity measured via the Minkowski metrics in a conceptual space or via DISTANCE in the semantic network is symmetric. Feature models are based on comparison of feature sets, which is in principle symmetric. The calculation results in an asymmetric similarity, if the set of features applying to concept $a$, but not to $b$ are valued differently to features applying to concept $b$, but not to $a$. Thus, similarity is asymmetric if the weighting factors $\alpha$ and $\beta$ for the difference sets $A-B$ and $B-A$ are not equal (equations 2 and 3 ). In an information retrieval task it is important that data source concepts meet the requirements of the query concept, but not the other way around. The hybrid similarity model accounts explicitly for this characteristic of retrieval tasks by using the conceptual space and relations of the query as basis. Geometric and network models cannot account for this requirement of retrieval tasks. For feature models, suitable values for $\alpha$ and $\beta$ must be determined. 
A HYBRID SIMILARITY MEASURE 31

\section{Summary and Conclusions}

We have proposed a hybrid semantic similarity measure to determine relevant concepts in spatial data retrieval. The hybrid approach combines conceptual spaces and semantic networks to describe the semantics of concepts including their properties as well as their relations to other concepts.

The main challenge of this similarity measure is the computation of semantic distance, which includes properties as well as relations. Concepts modeled as conceptual regions are the second difficulty faced: traditional similarity measures of conceptual spaces reduce concepts to a single instance to measure the semantic distance. To avoid this information loss, a similarity measure accounting for the whole conceptual regions is required. The hybrid similarity measure first reduces the calculation to one-dimensional problems, the dimension-wise intervals are compared and a distance value is determined, which is consolidated to a single semantic distance using the Minkowski metrics. The hybrid similarity measure accounts for asymmetric similarity due to context variations via different significance of dimensions and relations. Moreover, it can be naturally extended to account for relations. The semantic distance between the related concepts is computed and integrated as a virtual dimension in the conceptual space.

The evaluation demonstrates that the hybrid similarity measure is very effective for semantic information retrieval. The effectiveness of the retrieval system is measured in terms of its precision and recall with graded relevance. The comparison of similarity measures provides clear evidence that our hybrid similarity measure is more suitable for the retrieval of spatial data than other existing semantic similarity measures.

The major problem of applying and evaluating semantic similarity measures is a general lack of semantic descriptions of spatial data sources. Currently only very few spatial data sources provide a description of their content and these descriptions are often limited to textual definitions of geographic feature types. These textual descriptions are far too imprecise and incomplete to use for an information retrieval system. In this evaluation, an expert was required to construct a comprehensive and structured semantic description. However, there is a general need construct comprehensive formal semantic specifications of spatial data sources (such as ontologies) (Probst, 2008) to enable semantic applications.

Also with respect to the similarity measure itself further work is required: The presented similarity measure deals with the retrieval at a conceptual level only. This is the level where semantic interoperability problems caused by different conceptualizations occur. Future work has to extend the retrieval method to the instance level and connect conceptual with instance information. 


\section{SCHWERING, KUHN}

\section{Acknowledgement}

Discussions with the members of the Muenster Semantic Interoperability Lab (MUSIL) and Ordnance Survey have greatly influenced the ideas presented here. The comments from three anonymous referees provided useful suggestions to improve the content of the paper. Funding from the University of Muenster and the Ordnance Survey of Great Britain is gratefully acknowledged.

\section{A Appendix}

The interval arithmetic used for the comparison of intervals is defined as follows: The length $l$ of an interval $I=[a, b]$ is defined by $l=b-a$.

The total length $l$ of several intervals $I_{i}=\left[a_{i}, b_{i}\right]$ is defined by $l=\sum_{i=1}^{n}\left(b_{i}-a_{i}\right)$. The intersection of two intervals $I_{1}=\left[a_{1}, b_{1}\right]$ and $I_{2}=\left[a_{2}, b_{2}\right]$ is defined by $I_{1} \cap I_{2}=\left\{\begin{array}{l}{\left[\max \left(a_{1}, a_{2}\right), \min \left(b_{1}, b_{2}\right)\right], \quad a_{1} \leq b_{2} \wedge b_{1} \geq a_{2}} \\ \emptyset \quad \text { otherwise }\end{array}\right.$

The difference interval of two intervals $I_{1}=\left[a_{1}, b_{1}\right]$ and $I_{2}=\left[a_{2}, b_{2}\right]$ is defined by $I_{1} \mid I_{2}=\left\{\begin{array}{l}{\left[a_{1}, \min \left(a_{2}, b_{1}\right)\right], \quad a_{1}<a_{2} \wedge b_{1} \leq b_{2}} \\ {\left[\max \left(a_{1}, b_{2}\right), b_{1}\right], \quad a_{1} \geq a_{2} \wedge b_{1}>b_{2}} \\ {\left[a_{1}, a_{2}\right] ;\left[b_{2}, b_{1}\right], \quad a_{1}<a_{2} \wedge b_{1}>b_{2}} \\ \emptyset, \quad \text { otherwise }\end{array}\right.$

The subtraction of two intervals $I_{1}=\left[a_{1}, b_{1}\right]$ and $I_{2}=\left[a_{2}, b_{2}\right]$ is defined by $I_{1}-I_{2}=\left[a_{1}-b_{2}, b_{1}-a_{2}\right]$

\section{References}

Attneave, F. (1950). Dimensions of similarity. American Journal of Psychology, $63,516-556$.

Collins, A. M., \& Quillian, M. (1969). Retrieval time from semantic memory. Journal of Verbal Learning and Verbal Behavior, 8, 240-247.

Devore, J., \& Peck, R. (2001). Statistics - the exploration and analysis of data (4th ed.). Pacific Grove, CA: Duxbury.

Fahrmeir, L. (2004). Statistik (5th ed.). Berlin, Germany: Springer.

Falk, D., Brill, D., \& Stork, D. (1986). Seeing the light - optics in nature, photography, color, vision and holography. New York, USA: Harper \& Row.

Gärdenfors, P. (2000). Conceptual spaces: The geometry of thought. Cambridge, MA: MIT Press. 
A HYBRID SIMILARITY MEASURE 33

Gentner, D., \& Landers, R. (1985). Analogical reminding: A good match is hard to find. In Proceedings of the international conference on systems, man and cybernetics.

Gentner, D., \& Markman, A. B. (1994). Structural alignment in comparison: No difference without similarity. Psychological Science, 5(3), 152-158.

Gentner, D., \& Markman, A. B. (1995). Similarity is like analogy: Structural alignment in comparison. In C. Cacciari (Ed.), Similarity in language, thought and perception (p. 111-147). Brussels, Belgium: Brepols.

Gentner, D., Rattermann, M. J., \& Forbus, K. D. (1993). The roles of similarity in transfer: Separating retrievability from inferential soundness. Cognitive Psychology, 25, 524-575.

Goldstone, R. L. (1994). Similarity, interactive activation, and mapping. Journal of Experimental Psychology: Learning, Memory, and Cognition, 20(1), 328.

Goldstone, R. L., \& Medin, D. L. (1994). Similarity, interactive activation and mapping: An overview. In J. Barnden \& H. K. (Eds.), Advances in connectionist and neural computation theory (Vol. 2: Analogical Connections, p. 321-362). New Jersey: Ablex.

Goldstone, R. L., \& Son, J. (2005). Similarity. In K. Holyoak \& R. Morrison (Eds.), Cambridge handbook of thinking and reasoning (p. 13-36). Cambridge: Cambridge University Press.

Hahn, U., Richardson, L. B., \& Chater, N. (2003). Similarity as transformation. Cognition, 87, 1-32.

Imai, S. (1977). Pattern similarity and cognitive transformations. Acta Psychologica, 41, 433-447.

Janowicz, K. (2006a). Towards a similarity-based identity assumption service for historical places. In M. Raubal, H. Miller, A. Frank, \& M. Goodchild (Eds.), 4th international conference on geographic information science (GIScience) 2006 (Vol. 4197, p. 199-216). Muenster; Germany: Springer.

Janowicz, K. (2006b). Sim-DL: Towards a semantic similarity measurement theory for the description logic ALCNR in geographic information retrieval. In R. Meersman, Z. Tari, \& P. Herrero (Eds.), 2nd international workshop on semantic-based geographical information systems (SeBGISO6) (Vol. 4278, p. 1681-1692). Montpellier, France: Springer.

Johannesson, M. (2002). Geometric models of similarity. Unpublished doctoral dissertation, Lund University. 


\section{SCHWERING, KUHN}

Li, B., \& Fonseca, F. (2006). Tdd: A comprehensive model for qualitative spatial similarity assessment. Spatial Cognition and Computation, 6(1), 31-62.

Markman, A. B. (1999). Knowledge representation (Vol. 1). Mahwah, New Jersey: Lawrence Erlbaum Associates.

Masolo, C., Borgo, S., Gangemi, A., Guarino, N., \& Oltramari, A. (2003). Wonderweb deliverable d18 (Tech. Rep. No. Del18). Laboratory for Applied Ontology - ISTC - CNR.

Melara, R. D., Marks, L. E., \& Lesko, K. E. (1992). Optional processes in similarity judgments. Perception \& Psychophysics, 51(2), 123-133.

Meyer, D. E. (1970). On the representation and retrieval of stored semantic information. Cognitive Psychology, 1, 242-300.

Probst, F. (2008). Observations, measurements and semantic reference spaces. Special Issue: Ontological Foundations for Conceptual Modeling. Journal of Applied Ontology., 3(1-2), 63-89.

Rada, R., Mili, H., Bicknell, E., \& Blettner, M. (1989). Development and application of a metric on semantic nets. IEEE Transactions on systems, man, and cybernetics, 19(1), 17-30.

Raubal, M. (2004). Formalizing conceptual spaces. In A. Varzi \& L. Vieu (Eds.), Formal ontology in information systems, 3rd international conference (fois 2004) (Vol. 114, p. 153-164). Torino, Italy: IOS Press.

Rodriguez, A. (2000). Assessing semantic similarity among spatial entity classes. Unpublished doctoral dissertation, University of Maine.

Rodriguez, A., \& Egenhofer, M. J. (2003a). Determining semantic similarity among entity classes from different ontologies. IEEE Transactions on Knowledge and Data Engineering, 15(2), 442-456.

Rodriguez, A., \& Egenhofer, M. J. (2003b). Determining semantic similarity among entity classes from different ontologies. IEEE Transactions on Knowledge and Data Engineering, 15(2), 442-456.

Rodriguez, A., \& Egenhofer, M. J. (2004). Comparing geospatial entity classes: An asymmetric and context-dependent similarity measure. International Journal of Geographical Information Science, 18(3), 229-256.

Sattath, S., \& Tversky, A. (1987). On the relation between common and distinctive feature models. Psychological Review, 94(1), 16-22.

Schaeffer, B., \& Wallace, R. (1970). Semantic similarity and the comparison of word meaning. Journal of Experimental Psychology: General, 86, 144-152. 
A HYBRID SIMILARITY MEASURE 35

Schwering, A. (2005). Hybrid model for semantic similarity measurement. In R. Meersman \& Z. Tari (Eds.), 4th international conference on ontologies, databases, and applications of semantics (ODBASE05) (Vol. 3761, p. 14491465). Agia Napa, Cyprus: Springer.

Schwering, A. (2007a). Evaluation of a semantic similarity measure for natural langugage spatial relations. In 5th conference on spatial information theory (COSIT07) (Vol. 4736, p. 116-132). Melbourne, Australia: Springer.

Schwering, A. (2007b). Semantic similarity measurement including spatial relations for semantic information retrieval of geo-spatial data. Solingen, Germany: Verlag Natur \& Wissenschaft.

Schwering, A. (2008). Approaches to semantic similarity measurement for geospatial data - a survey. Transactions in GIS, 12(1), 5-29.

Schwering, A., \& Raubal, M. (2005). Spatial relations for semantic similarity measurement. In J. Akoka, S. W. Liddle, I.-Y. Song, M. Bertolotto, I. Comyn-Wattiau, W.-J. vanden Heuvel, M. Kolp, J. Trujillo, C. Kop, \& H. C. Mayr (Eds.), 2nd international workshop on conceptual modeling for geographic information systems (CoMoGIS2005) (Vol. 3770, p. 259-269). Klagenfurt, Austria.: Springer.

Shepard, R. N. (1957). Stimulus and response generalization: A stochastic model relating generalization to distance in psychological space. Psychometrika, 22(4), 325-345.

Shepard, R. N. (1958a). Stimulus and response generalization: Deduction of the generalization gradient from a trace model. Psychological Review, 65(4), 242-256.

Shepard, R. N. (1958b). Stimulus and response generalization: Tests of a model relating generalization to distance in psychological space. Journal of Experimental Psychology, 55(6), 509-523.

Suppes, P., Krantz, D. M., Luce, R. D., \& Tversky, A. (1989). Foundations of measurement - geometrical, threshold, and probabilistic representations (Vol. 2). San Diego, California, USA: Academic Press, Inc.

Torgerson, W. S. (1958). Theory and methods of scaling. New York: Wiley.

Torgerson, W. S. (1965). Multidimensional scaling of similarity. Psychometrika, 30(4), 379-393.

Tversky, A. (1977). Features of similarity. Psychological Review, 84(4), 327-352. 


\section{SCHWERING, KUHN}

Tversky, A., \& Gati, I. (1978). Studies of similarity. In E. Rosch \& B. Lloyd (Eds.), Cognition and categorization (p. 79-98). Hillsdale, NJ: Lawrence Erlbaum.

Tversky, A., \& Gati, I. (1982). Similarity, separability, and the triangle inequality. Psychological Review, 89(2), 123-154.

Wiener-Ehrlich, W. K., Bart, W. M., \& Millward, R. (1980). An analysis of generative representation systems. Journal of Mathematical Psychology, 21(3), 219-246.

Wyszecki, G., \& Stiles, W. S. (2000). Color science - concepts and methods, quantitative data and formulae (2nd ed.). New York, USA: Wiley. 